\title{
The Research and Implementation of Field Data Acquisition System in the Southern Plateau Based on the Android Platform
}

\author{
Yang Yang ${ }^{*}$, Hou Guang-liang, Yin Qing-Jun , and Wang Qing-bo, \\ School of Life and Geographic Science Qinghai Normal University Xining, China \\ *Corresponding author: Yang Yang, 51701778@qq.com
}

\begin{abstract}
Based on the Android platform, we have developed a set of basic mobile GIS software platform, built a integrated service platform which is composed of positioning, analysis, overlay compliance judgment, site selection and statistics, and obtained the real-time spatial data, so as to improve the efficiency of the work and the management of field data acquisition and precision level, provide effective means of auxiliary for the efficient and accurate data acquisition, data management and scientific decision.
\end{abstract}

Keywords: component, formatting, android platform, real-time spatial data, field data acquisition

\section{Introduction}

Mobile GIS is the geographic information system based on mobile computing environment, runs on mobile devices, and has some part main function of the desktop GIS [1]. Due to the rapid expansion of the large number of new technology of mobile GIS, such as mobile access technology, mobile access method, mobile terminals, mobile GIS has been able to provide real-time spatial information "4 A" service [2].

Based on mobile GIS platform construction, it is closely combined with the field data acquisition work in southern plateau of Qinghai, makes full use of modern wireless communication technology, 3S integration technology, mobile positioning technology, etc., provides work management, locating information query, field monitoring data collection in the Qinghai lake site, etc. [3], which is the main auxiliary tools of staff, and it has great significance to promote the field monitoring work in southern Qinghai plateau.

\section{The System Structure of Mobile GIS}

The logic bottom of MOBILE GIS software platform is embedded operating system, such as Android, iOS and WINDOWS MOBILE, etc.; The layer above it is a spatial data management module, which manages mobile GIS spatial data, and is responsible for synchronous transmission with desktop GIS or WEBGIS data; Layer is on top of mobile GIS platform attribute query, projection transformation and GPS integration and so on, the basic function modules, including spatial data visualization, the layer of each module can according to need to cut or increase; The top layer is geared to the needs of users of all kinds of mobile GIS service software.

\section{Android Platform}

Android, is an Open source mobile devices based on Linux operating system [4], it is mainly used for smart phones and tablets, which is leaded and founded by the Open Handset Alliance (OHA) persistently. Android has released the latest version, which is Android 5.0 [5]. 
Android is composed of $\mathrm{x}$ the Linux kernel, system, application framework, and application, based on Linux kernel, open source code "Fig. 1".

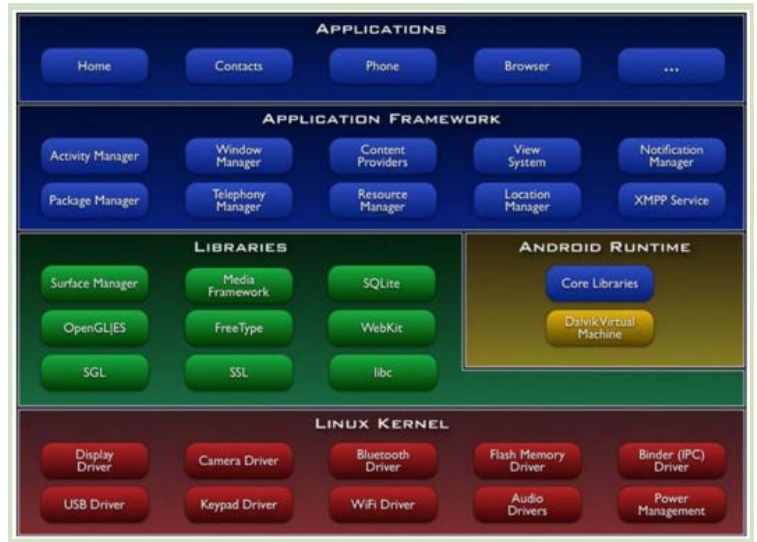

Fig. 1- The composition and structure of Android.

ArcGIS Runtime SDKs for Smartphones and Tablets is the mobile application development kit Esri for developers, currently supports three mainstream mobile operating system which are iOS, Android, Windows Phone. ArcGIS Runtime SDK for Android gets the data and services by ArcGIS Server REST.

\section{The System Overall Frame}

Program includes calls, text messaging program, etc., application software is self-exploitation, written by Java [6]. This platform conducts software design based on the Android operating system, it is because of the openness of the middleware development at the bottom, at present gets support from numerous brand smartphones and handheld tablet, combined with cooperation between mobile communication operators and smartphone manufacturers, do not need to expend energy to research and develop hardware platform, operating system, and the middle function library, and directly focus the main energy on the development of GIS platform application software for mobile field data collection [7], the architecture diagram is shown in "Fig.2".

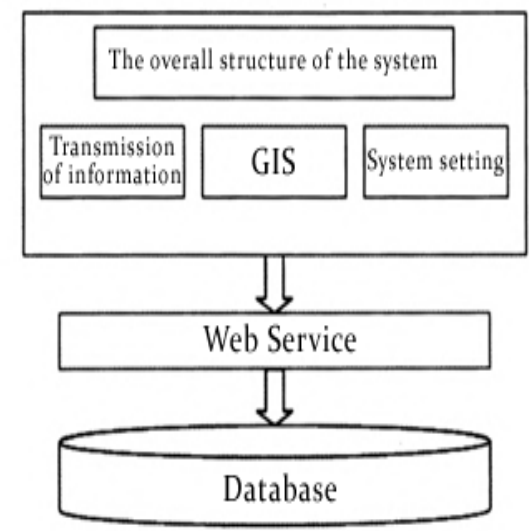

Fig. 2- The system's overall framework.

\subsection{The system composition}

The purpose of this system is the use of computer technology, network, GPS, RS and GIS technologies etc. [8], builds a comprehensive business mobile platform which is composed of positioning, planning, analysis, overlay compliance judgment, site selection and statistics, thus greatly improves the work efficiency, reduces the repeated operation and complexity due to 
subjective location or judge, to realize real-time and accurate programme conditions, improve the level of office management. Provide efficient and convenient services for real-time management of leaders at different levels, and provide effective auxiliary means for scientific decision [9]. In the whole system architecture, the use of the mixed solution which are C/S and M/S, fully meets the demands of the users of different levels and functions. Under the C/S structure, the client uses the NET development, GIS platform is all types of desktop geographic information system platform [10], product, analyze, debug and the registrate the data of the platform, and configurate and manage M/S system, some parts of function mainly aim at the platform administrator and privileged users. Under the M/S structure, conduct mobile office on-site by using the intelligent terminal based on Android, obtain real-time location data, this function is applicable to the general users [11].

\subsection{The system environment}

The operating system: the Android 4.1 or above.

Hardware configuration: Android tablets whose memory is $512 \mathrm{MB}$, built-in GPS, 1GB memory card.

Development environment configuration: the construction method of the software development environment, WindowsXPSP3 or below the Windows 7 operating system, firstly need to install the JDK, then download and install the Eclipse IDE, install the ADT plugin, download and set up the SDK; In order to obtain the location information, also chose GPS positioning module that supports platform “Fig.3”.

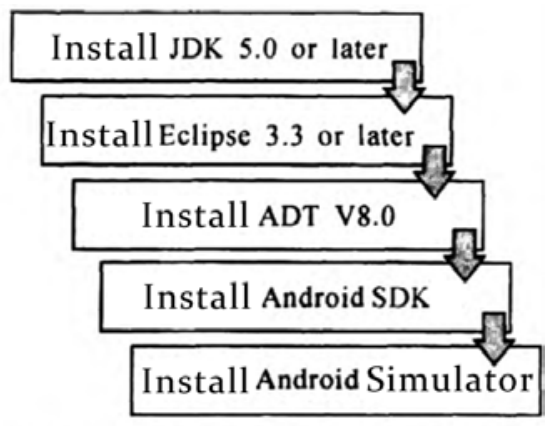

Fig. 3 - The procedure chart of configuration of development environment of Android.

\section{The Platform Implementation Based on Mobile GIS}

\subsection{The realization of the mobile terminal platform with service management}

The mobile data collection GIS platform based on Android can easily realize real-time location, site trial and judgment of planning conformance, monitoring site investigation, mainly includes maps, field management (including my position, designated spot), distance measurement, area measurement, planning and compliance analysis, interest management, etc., also includes amplification, narrow, roaming, operation is similar to smart phones Android/tablet.

My position: locate my position, click "my location" button, under the premise of GPS signals, it can locate the user's current position, can look up the coordinates of the data on the lower left corner of screen, avoid to lookup reference substance on the field, unable to know the location of the situation, and can judge oneself of position through the compass upper right corner.

Specified location: locate " designated spot ", this function is applicable to the users themselves know want to know the location of the coordinates, click on the "position" button, you can enter the 
latitude and longitude (minutes) way to pinpoint the location, the known coordinates, view the site condition, and through the upper right corner of a compass can judge oneself of position.

Distance measurement, amplification, narrow or roaming the appropriate screen position, first click on the "distance measurement" button, click want to measure the line on the screen, click on the measurement, and finally click "calculate" button to go to know the distance measurement; Click "clear" to clear point trajectory and the measurement results.

Area measurement: amplification, narrow or roaming the appropriate screen position, first click on the area of measurement button, click want to measure the area on the screen, click on the measurement, and finally click the "calculate" button know measured area; Click "clear" to clear point trajectory and the measurement results.

Planning of conformity analysis (superposition analysis): analysis function is an important module of this system, conformity judgment in the planning, analysis, and have important applications on the site. During the field site operations, for example, by GPS positioning to the current position first, preliminary determined when before, by switching map, switch to the image, you can click on the area of measurement button, measuring $\mathrm{Bi}$ location area and the measured line trajectory is in right now, you can switch the map, such as sheet, see the site planning of compliance situation, through the different colors and airspaces' illustrations can probably know something about the conformity planning, avoid the repeated, site work and real-time access to land area and land types, greatly facilitate the work of the planning and site selection analysis.

Index figure: after entering Huiyang district mobile monitoring platform, will see the "index", when you reach to examine after the project is located, click on the top left corner of the screen with your finger "my location" button, you can know what is the current of the evaluated integratedly (development zone), click on the corresponding evaluated integratedly (development zone) to enter the name of the corresponding area, can begin to look at the picture.

Returns: used to exit the current evaluated integratedly (development zone), to choose. Other evaluated integratedly (development zone), seeing other evaluated integratedly (development zone) of land and project situation.

\subsection{The characteristic of platform}

Mobility: running on all kinds of mobile terminal, and interact with the server through wireless communication data real-time access to space, can undertake spatial information services anytime and anywhere, can also be out of the constraints of server and transmission medium run independently, with mobility.

Client diversity: the client of mobile GIS is used outdoors mobile terminal equipment, its range is wide, can be a powerful computing ability is the mainstream of the micro computer, can also be a smaller screen, all kinds of mobile computing terminal functional limitations, such as PDA, mobile phone, etc., can even be a dedicated embedded GIS equipment, which determine the mobile GIS is supposed to be an open platform for the scalable (without special GIS platform).

Dynamic (real-time): as a kind of application service system, should be able to respond in a timely manner to the user's request, can deal with the user real-time change over time in the environment factors, in the process of moving, not restricted to collected timely treatment and publish the relevant information to the user.This is one of the biggest characteristic of mobile GIS. 
Operation is simple: by smart devices to operate, easy to use, easy to carry, to avoid the field data acquisition work carry paper maps of trouble, but also can make real-time overlay, convenient and simple.

\section{Conclusions}

Through the images of to look at the scene, and calculate its area; Then quickly switch to the plan immediately, the general land use planning; Then look at the land use present situation, judge its update change situation; Finally to switch to other related figure, such as city sheet, see its compliance situation, so the whole site location in a short period of time can immediately get a preliminary results, avoid the repeated work, planning on the conformity to the user a good efficient, scientific decision-making impression. But in actual development process, this platform is not based on any platform of GIS and other engines, completely independent development, so for the mass data processing, has always been one of the key technologies in the project; how to solve this problem? For huge amounts of data, this project USES is subregional slice method, although not completely and clear as the original image exactly, but also can satisfy the current preliminary user requirements; Another key technical problems is the problem of data coordinate registration, the project USES is more coordinate configuration, but for different areas, using the method of acquisition to configure local landmark for large area is a little error, hope a breakthrough in the subsequent research. Practice has proved that the fusion of GIS, GPS, RS and mobile supervisory wide to get figure of mobile GIS platform for the development of completed and put into use, to solve the land, planning, environmental protection and geological disaster survey and field with figure survey work such as convenience and real-time performance, meet the land, planning and environmental management in areas such as field actual need, has realized the land, planning, environmental protection field paperless, facilitation and real-time, scientific decision analysis, become its day-to-day business operation system technology foundation, has made remarkable application benefits, users get the praise. But the system in such aspects as precise positioning and data comprehensive integration method and need to be further studied and discussed, the data maintenance mechanism remains to be further established.

\section{Acknowledgment}

The authors will thank Hou Guang-liang for his great help in this paper. This paper is supported by Project of Natural Science Foundation of Qinghai province (2013-Z-914).

\section{REFERENCES}

1. M. RETO, Android High-level programming, Beijing, Tsinghua university press,2010.

2. E. BURNETrE, Android based tutorial, Beijing, people's posts and telecommunications publishing house, 2009 .

3. C. Zhang, A.C. Wang, and Z. Wang, The study on mobile phone software based on J2ME and J2EE, Journal of heilongjiang science and technology information, 2007, 3: 21.

4. S.L. Gai, Android development guide, Beijing, people's posts and telecommunications publishing house, 2010.

5. B.G. Liu, The design and optimization of Android communication module, Wuhan, wuhan university of technology, 2010. 
6. X.H. Shu, The service design of mobile Web map based on Andwid platform, Dalian, dalian maritime university, 2009.

7. J.C. Lin, and D.Y. Hu, Java typical application investigate 1000 cases-database application foundation, Beijing, the group electronic publishing house, 2009.

8. H.Z. Guo, Andwid application development explanation, Beijing, electronic industry press, 2010.

9. Y. Qian, The design of the terminal power wireless communication scheme based on Android, Journal of electric power, vol. 26 (1) (2011) 6063 .

10. J.X. Zhao, The development of mobile location services based on the Android platform, The development and implementation of modern trade industry, vol. 22 (20) (2010) 271-273

11. L.P. Nong, L.H. Wang, and Y.P. Huang, The research of Android's applications in embedded navigation system, Computer engineering and design, vol. 31 (11) (2010) 2473-2476. 